\title{
Der Placeboeffekt ist sehr groß
}

Fragestellung: Sind die Nicht-Benzodiazepine Eszopiclon, Zaleplon und Zolpidem (Z-Substanzen) wirksamer als Placebo, wenn bei einer Metaanalyse auch Daten aus Studien hinzugezogen werden, die nicht publiziert wurden?

Hintergrund: Ziel der Metaanalyse war es, die Wirksamkeit der Z-Substanzen Eszopiclon, Zaleplon und Zolpidem im Vergleich zu Placebo zu untersuchen und hierbei die Auswirkungen von Publikationsfehlern zu minimieren. Dazu wurde auf eine Datenbank der US Food and Drug Administration (FDA) zugegriffen, in die pharmazeutische Unternehmen im Rahmen von Arzneimittelzulassungen Daten aus allen von ihnen finanzierten veröffentlichten und unveröffentlichten Studien zur Verfügung stellen müssen.

Patienten und Methodik: Die Metaanalyse schloss 13 klinische Studien mit insgesamt 4.378 Teilnehmern ein, die in doppelblinMiddlemass J et al. Effectiveness of non-benzodiazepine hypnotics in treatment of adult insomnia: meta-analysis of data submitted to the Food and Drug Administration. BMJ 2012; 345: e8343.
Huedo-Medina TB, Kirsch I, dem placebokontrollierten Design oben genannte ZSubstanzen bei primärer Insomnie untersucht hatten. Die primären Endpunkte waren die polysomnographisch ermittelte und subjektive Schlaflatenz, sekundäre Endpunkte unter anderem polysomnografisch ermittelte und subjektive Gesamtschlafdauer, Anzahl und Dauer von Wachphasen und die Schlafeffizienz, wobei Daten zu den sekundären Endpunkten nur aus wenigen der eingeschlossenen Studien extrahiert werden konnten, sodass sich hieraus keine statistisch verwertbaren Ergebnisse ergaben. Die Behandlungsdauer in den eingeschlossenen Untersuchungen war mindestens 14 und höchstens 180 Tage.

Ergebnisse: Für die primären Endpunkte der Schlaflatenzen zeigte die Analyse der gewichteten standardisierten mittleren Differenz sowohl für Placebo (polysomnografisch: $-0,39$, $95 \%$-Konfidenzintervall [KI] $-0,54$ bis $-0,23$; subjektiv: $-0,33$; $95 \%-\mathrm{KI}-0,63$ bis $-0,03$ ) als auch die Z-Substanzen (polysomnografisch: $-0,93$; $95 \%$-KI $-1,32$ bis $-0,54$; subjektiv: $-0,67$; $95 \%$ KI $-1,30$ bis -0,03) signifikante verkürzende Effekt. Bei einem Vergleich zwischen Placebo und den Z-Substanzen ergaben sich ebenfalls signifikante, aber kleine bis mittlere Unterschiede in der polysomnografisch ermittelten und subjektiven Schlaflatenz zwischen Verum und Placebo. Umgerechnet bedeutet das, dass Nicht-Benzodiazepin-Hypnotika die Einschlaflatenz im Vergleich zu Placebo um 22 Minuten, in Kombination mit einem Placeboeffekt um 42 Minuten verkürzen.

Schlussfolgerungen: Die Autoren folgern, dass Z-Substanzen objektiv und subjektiv die Schlaflatenz verkürzen, aber etwa die Hälfte der Wirksamkeit durch den Placeboeffekt erklärt wird.

\section{Augenmerk stärker auf psychologische Interventionen richten}

Die Metaanalyse zeigte einen Effekt von drei Z-Substanzen auf die Schlaflatenz, der aber im Vergleich zum Placeboeffekt klinisch eher klein zu sein scheint. Interessant ist, dass auch unveröffentlichte Daten in die Analyse eingeschlossen wurden. Ein gewisser Schwachpunkt ist, dass nur zu den primären Endpunkten statistisch verwertbare Aussagen getroffen werden konnten, verschiedene Medikamente (mit sehr unterschiedlichen Halbwertzeiten) und Dosierungen in „einen Topf" geworfen wurden und keine Auswertung von unerwünschten Wirkungen erfolgte.

Für die primären Insomnien hat sich eine kognitiv-verhaltenstherapeutische Therapie als besonders wirkungsvoll und in einem kürzlich publizierten systematischen Review den Benzodiazepinen in der Langzeitbehandlung überlegen gezeigt [1]. Demgegenüber steht die klinische Erfahrung, dass viele insomnische Patienten dauerhaft mit Hypnotika behandelt werden. Die Verschreibung von Benzodiazepinen zur pharmakologischen Behandlung von Insomnien hat zwar abgenommen, der Gebrauch von Hypnotika insgesamt ist aber durch die Markteinführung der Z-Substanzen unverändert hoch geblieben. Dies mag auch darin begründet sein, dass die Z-Substanzen in der Wahrnehmung von Hausärzten sicherer und effektiver als Benzodiazepine sind [2], wofür es keine über- zeugende Evidenz gibt. Vor diesem Hintergrund ist diese Metaanalyse von großem Interesse, spricht sie doch dafür, dass ein nicht unerheblicher Teil der Wirkung der Z-Substanzen durch einen Placeboeffekt vermittelt wird. Dies unterstreicht, dass weiterhin eine erhöhte Aufmerksamkeit auf psychologische Interventionen für Schlaflosigkeit gerichtet sein sollte und die Pharmakotherapie nur ein Baustein in einem therapeutischen Gesamtkonzept sein darf.
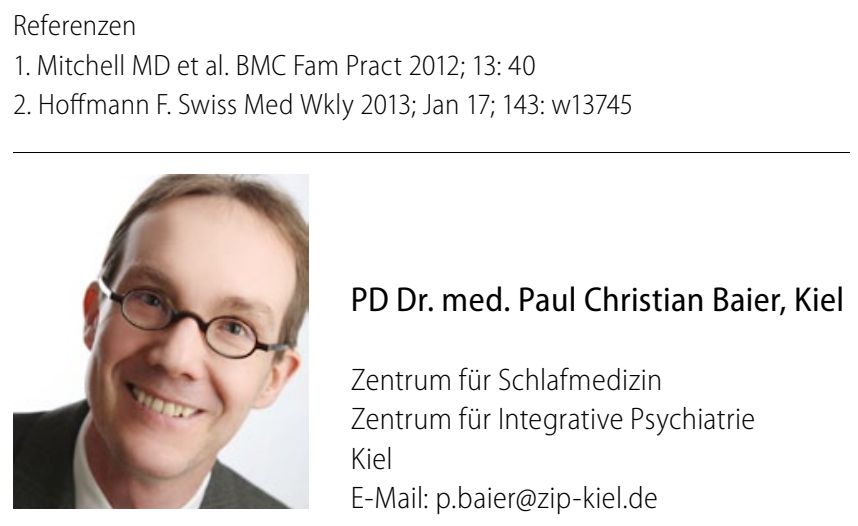